\title{
Correlation Functions for $\beta=1$ Ensembles of Matrices of Odd Size
}

\author{
Christopher D. Sinclair*
}

October 26, 2018

\begin{abstract}
Using the method of Tracy and Widom we rederive the correlation functions for $\beta=1$ Hermitian and real asymmetric ensembles of $N \times N$ matrices with $N$ odd.
\end{abstract}

\section{Introduction}

The starting point for many results concerning the spectral theory of random matrices is the derivation of a determinental or Pfaffian form for the correlation functions of the eigenvalues. Perhaps the most well-studied ensembles are Hermitian ensembles having joint probability density function (JPDF) of the form

$$
\Omega(\boldsymbol{\lambda})=\frac{1}{Z}\left\{\prod_{n=1}^{N} w\left(\lambda_{n}\right)\right\}|\Delta(\boldsymbol{\lambda})|^{\beta}
$$

where $Z$ a normalizing constant, $w: \mathbb{R} \rightarrow[0, \infty)$ is a weight function and $\Delta(\boldsymbol{\lambda})$ is the $N \times N$ Vandermonde determinant in the coordinates of $\boldsymbol{\lambda}$. The parameter $\beta$ is called the inverse temperature parameter due to its physical interpretation in the study statistics of log-gases, another area where joint densities of the form (1.1) arise. When $w$ is Gaussian and $\beta=1,2$ or $4, \Omega$ is the JPDF of the ensemble of $N \times N$ real symmetric $(\beta=1)$, complex Hermitian $(\beta=2)$ and self-dual $(\beta=4)$ matrices, the independent entries of which are chosen with Gaussian density. The details of this derivation are given in [15] and the interpretation of $\Omega$ in the study of log-gases is given in [8]. We will denote the ensemble with joint density $\Omega$ by $w \beta \mathrm{E}$.

The $n$th correlation function of $w \beta \mathrm{E}$ is defined by

$$
R_{n}(\boldsymbol{\lambda})=\frac{1}{(N-n) !} \int_{\mathbb{R}^{N-n}} \Omega(\boldsymbol{\lambda} \vee \boldsymbol{\xi}) d \mu^{N-n}(\boldsymbol{\xi}) ; \quad n=1,2 \ldots, N,
$$

where $\boldsymbol{\lambda} \vee \boldsymbol{\xi}=\left(\lambda_{1}, \ldots, \lambda_{n}, \xi_{1}, \ldots, \xi_{N-n}\right)$ and $\mu$ is Lebesgue measure on $\mathbb{R}$. That is, after renormalization, $R_{n}$ gives the $n$th marginal probability density of $\Omega$.

When $\beta=2$, Fubini's Theorem together with elementary row and column operations on the Vandermonde determinant in the integrand lead to the determinental formula

$$
R_{n}(\lambda)=\operatorname{det}\left[K_{N}\left(\lambda_{j}, \lambda_{k}\right)\right]_{j, k=1}^{n},
$$

${ }^{*}$ This research was supported in part by the National Science Foundation (DMS-0801243) 
where $K_{N}$ is the kernel of a certain operator on $L^{2}(w d \mu)$.

Following pioneering work of Dyson [6], Mehta derived a Pfaffian ${ }^{1}$ form for the correlation functions of the Gaussian $\beta=1$ and 4 Hermitian ensembles [14]. This was repeated for general weights by Mehta and Mahoux [13], except for the case $\beta=1$ and $N$ odd. This last remaining case was given by Adler, Forrester and Nagao [1]. In each of these cases, the Pfaffian formulation of $R_{n}$ is given by

$$
R_{n}(\lambda)=\operatorname{Pf}\left[K_{N}\left(\lambda_{j}, \lambda_{k}\right)\right]_{j, k=1}^{n}
$$

where here, $K_{N}$ is a $2 \times 2$ matrix which is the kernel of an operator on $L^{2}(w d \mu) \times L^{2}(w d \mu)$. (The matrix $K_{N}$ depends on $\beta$ and has a slightly different structure depending on whether $N$ is even or odd).

Much of the interest in the spectral theory of random matrices revolves around the eigenvalue statistics as $N \rightarrow \infty$. It is for this reason that the determinental/Pfaffian formulations for the correlation functions are so important: $N$ appears as a parameter in the kernel and in many cases, $K_{N}$ be analyzed as $N \rightarrow \infty$. In contrast, the number of integrations necessary to arrive at the correlation functions via their definition, increases with $N$-a situation which is not as easily handled.

These determinental/Pfaffian forms of the correlation functions have since been derived by various different methods. Of particular note is the method of Tracy and Widom who, for all cases except $\beta=1, N$ odd, derive the determinant/Pfaffian forms of the correlation functions using the fact that, if $\mathbf{A}$ is a $T \times N$ and $\mathbf{B}$ is an $N \times T$ matrix, then

$$
\operatorname{det}(\mathbf{I}-\mathbf{A B})=\operatorname{det}(\mathbf{I}-\mathbf{B A}),
$$

where on the left hand side of this equation $\mathbf{I}$ is the $T \times T$ identity matrix, and on the right hand side it is the $N \times N$ identity matrix. (Equation (1.2) is more generally true; a fact which Tracy and Widom exploit to great advantage in a number of other situations). When $\beta=2$ this identity leads immediately to the determinental correlation functions whereas, when $\beta=1$ or $\beta=4$, the path from (1.2) to the Pfaffian correlation functions is more convoluted. The reason for this extra difficulty is that (1.2) is an identity about determinants, whereas what is really needed is an identity about Pfaffians. Tracy and Widom get around this difficulty by using the important observation that, if $\mathbf{A}$ is an antisymmetric square matrix of even size, then $\operatorname{det} \mathbf{A}=(\operatorname{Pf} \mathbf{A})^{2}$.

The extra circumlocutions necessary in the derivation of the correlation functions can be eliminated by using, in place of (1.2), the fact that

$$
\frac{\operatorname{Pf}\left(\mathbf{C}^{-\boldsymbol{\top}}-\mathbf{A}^{\top} \mathbf{B A}\right)}{\operatorname{Pf} \mathbf{C}^{-\top}}=\frac{\operatorname{Pf}\left(\mathbf{B}^{-\boldsymbol{\top}}-\mathbf{A} \mathbf{C} \mathbf{A}^{\top}\right)}{\operatorname{Pf} \mathbf{B}^{-\top}},
$$

where $\mathbf{B}$ and $\mathbf{C}$ are arbitrary antisymmetric $2 T \times 2 T$ and $2 N \times 2 N$ matrices with nonzero Pfaffians, and $\mathbf{A}$ is an arbitrary $2 N \times 2 T$ matrix. The derivation of the correlation functions using (1.3) in the case where $N$ is even was carried out by Borodin and Sinclair in $[4$, Appendix A]. Equation (1.3) is Rains' Pfaffian Cauchy-Binet formula [16]; a proof is given in $[4$, Appendix B].

The main purpose of this note is to show how (1.3) can be used to derive the Pfaffian form of the correlation functions in the case when $\beta=1$ and $N$ is odd, thus completing

\footnotetext{
${ }^{1}$ In fact Dyson, Mehta and many who followed, expressed the correlation functions in terms of Quaternion determinants, but in the more recent literature these are usually expressed in terms of Pfaffians.
} 
the work started by Tracy and Widom. We will also show how (1.3) can be used to derive the correlation functions of real asymmetric ensembles in the case when $N$ is odd. For $N$ even a derivation of the correlation functions of real asymmetric ensembles using (1.3) was given by Borodin and Sinclair [3]. The work here will complement the existing methods for deriving the correlation functions of real asymmetric ensemble given by Sommers and Wieczorek [20] and Mays and Forrester [9].

\section{$1.1 \quad$ Real Asymmetric Matrices}

Ginibre's real ensemble of $N \times N$ matrices is given by $\mathbb{R}^{N \times N}$ together with a probability measure specified by treating the entries of the matrices as independent standard normal random variables [11]. This ensemble is complicated by the fact that there are two species of eigenvalues: real and complex conjugate pairs. Among the implications of this is that there is no one JPDF for the ensemble. Instead, we have several partial JPDFs indexed by pairs non-negative integers $L$ and $M$ satisfying $L+2 M=N ; L$ represents the number of real eigenvalues in a 'sector' and $M$ the number of pairs of complex conjugate eigenvalues. These partial JPDFs are given by

$$
\Omega_{L, M}(\boldsymbol{\alpha}, \boldsymbol{\beta})=\frac{2^{M}}{Z}\left\{\prod_{\ell=1}^{L} w\left(\alpha_{\ell}\right) \prod_{m=1}^{M} w\left(\beta_{m}\right) w\left(\bar{\beta}_{m}\right)\right\}|\Delta(\boldsymbol{\alpha} \vee \boldsymbol{\beta} \curlyvee \overline{\boldsymbol{\beta}})|,
$$

where $Z$ is a normalizing constant (that depends on $N$ but not $L$ and $M), \Delta(\boldsymbol{\alpha} \vee \boldsymbol{\beta} \curlyvee \overline{\boldsymbol{\beta}})$ is the $N \times N$ Vandermonde determinant in the variables

$$
\alpha_{1}, \ldots, \alpha_{L}, \beta_{1}, \bar{\beta}_{1}, \ldots, \beta_{M}, \bar{\beta}_{M},
$$

and $w: \mathbb{C} \rightarrow[0, \infty)$ is a weight function given by

$$
w(\lambda)=e^{-\lambda^{2} / 2} \sqrt{\operatorname{erfc}(\sqrt{2}|\operatorname{Im}(\lambda)|)} .
$$

When $L=N$ and $M=0,(1.4)$ was established by Ginibre in his founding treatise on real asymmetric ensembles [11]. The general case was established three decades later independently by Lehmann and Sommers [12] and Edelman[7].

We may generalize Ginibre's real ensemble to other ensembles of real asymmetric matrices by allowing $w$ to be another function, and we call such an ensemble the real asymmetric ensemble with weight function $w$; here we will only consider weight functions satisfying $w(\lambda)=w(\bar{\lambda})$ for all $\lambda \in \mathbb{C}$.

Given non-negative integers $\ell$ and $m$ with $\ell+2 m \leq N$, we define the $\ell, m$-correlation function of the real asymmetric ensemble with weight function $w$ by $R_{\ell, m}: \mathbb{R}^{\ell} \times \mathbb{C}^{m} \rightarrow[0, \infty)$, where

$$
\begin{aligned}
R_{\ell, m}(\mathbf{x}, \mathbf{z})= & \sum_{L \geq \ell, M \geq m} \frac{1}{(L-\ell) !(M-m) ! 2^{M-m}} \\
& \times \int_{\mathbb{R}^{L-\ell}} \int_{\mathbb{C}^{M-m}} \Omega_{L, M}(\mathbf{x} \vee \boldsymbol{\alpha}, \mathbf{z} \vee \boldsymbol{\beta}) d \mu_{1}^{L-\ell}(\boldsymbol{\alpha}) d \mu_{2}^{M-m}(\boldsymbol{\beta}),
\end{aligned}
$$

where $\mu_{1}$ is Lebesgue measure on $\mathbb{R}$ and $\mu_{2}$ is Lebesgue measure on $\mathbb{C}$. 
One goal of the current manuscript is to show that, when $N$ is odd, $R_{\ell, m}(\mathbf{x}, \mathbf{z})$ can be written as

$$
R_{\ell, m}(\mathbf{x}, \mathbf{z})=\operatorname{Pf}\left[\begin{array}{ll}
K_{N}\left(x_{j}, x_{j^{\prime}}\right) & K_{N}\left(x_{j}, z_{k^{\prime}}\right) \\
K_{N}\left(z_{k}, x_{j^{\prime}}\right) & K_{N}\left(z_{k}, z_{k^{\prime}}\right)
\end{array}\right]_{\substack{j, j^{\prime}=1, \ldots, \ell \\
k, k^{\prime}=1, \ldots, m}},
$$

where $K_{N}$ is a particular $2 \times 2$ matrix kernel acting on $L^{2}\left(\mu_{1}+\mu_{2}\right) \times L^{2}\left(\mu_{1}+\mu_{2}\right)$, the exact nature of which will be explained in the sequel. A similar statement is true when $N$ is even $[3,4,19,10]$, and there are other existing (and arguably more complicated) methods for the $N$ odd case $[20,9]$.

\section{2 de Bruijn's Identities}

\subsection{For Hermitian Ensemble}

Given a measure $\nu$ on $\mathbb{R}$, we define

$$
Z_{N, \beta}^{\nu}=\int_{\mathbb{R}^{N}}|\Delta(\boldsymbol{\lambda})|^{\beta} d \nu^{N}(\boldsymbol{\lambda}) .
$$

When $d \nu=w d \mu$ for a function $w: \mathbb{R} \rightarrow[0, \infty), Z_{N, \beta}^{\nu}$ is the normalizing constant for the corresponding ensemble with weight function $w$. We call $Z_{N, \beta}^{\nu}$ the partition function of the corresponding ensemble.

A first step in the derivation of Pfaffian or determinantal form of the correlation functions for such ensembles is to write $Z_{N, \beta}^{\nu}$ as a determinant or a Pfaffian. This can only be done when $\beta=1,2$ or 4 . These identities, the first due to Andréief [2] and the second two to de Bruijn [5], can be formulated in our setting as follows: Suppose $p_{0}(\lambda), p_{1}(\lambda), \ldots$ are arbitrary monic polynomials such that $\operatorname{deg} p_{n}=n$, then

$$
\begin{gathered}
Z_{N, 2}^{\nu}=N ! \operatorname{det}\left[\int_{\mathbb{R}} p_{j}(\lambda) p_{k}(\lambda) d \nu(\lambda)\right]_{j, k=0}^{N-1}, \\
Z_{N, 4}^{\nu}=(2 N) ! \operatorname{Pf}\left[\int_{\mathbb{R}} p_{j}(\lambda) p_{k}^{\prime}(\lambda)-p_{k}(\lambda) p_{j}^{\prime}(\lambda) d \nu(\lambda)\right]_{j, k=0}^{2 N-1}
\end{gathered}
$$

and when $N$ is even

$$
Z_{N, 1}^{\nu}=\operatorname{Pf}\left[\int_{\mathbb{R}} \int_{\mathbb{R}} p_{j}(\lambda) p_{k}(\eta) \operatorname{sgn}(\eta-\lambda) d \nu(\eta) d \nu(\lambda)\right]_{j, k=0}^{N-1} .
$$

We define the $N \times N$ matrix $\mathbf{U}_{N, 1}^{\nu}$ by

$$
\mathbf{U}_{N, 1}^{\nu}=\left[\int_{\mathbb{R}} \int_{\mathbb{R}} p_{j}(\lambda) p_{k}(\eta) \operatorname{sgn}(\eta-\lambda) d \nu(\eta) d \nu(\lambda)\right]_{j, k=0}^{N-1} .
$$

Thus, when $N$ is even, $Z_{N, 1}^{\nu}=\operatorname{Pf} \mathbf{U}_{N, 1}^{\nu}$. One reason de Bruijn did not provide an identity for $Z_{N, 1}^{\nu}$ for odd $N$ is that the Pfaffian is only defined for antisymmetric square matrices with an even number of rows and columns. We may produce a de Bruijn identity in the odd 
$N$ case by suitably altering the matrix to appear on the right hand side of the expression for $Z_{N, 1}^{\nu}$. Specifically, for $N$ odd, we have $Z_{N, 1}^{\nu}=\operatorname{Pf} \mathbf{W}_{N, 1}^{\nu}$, where

$$
\mathbf{W}_{N, 1}^{\nu}=\left[\begin{array}{cccc} 
& & \int_{\mathbb{R}} p_{0}(\lambda) d \nu(\lambda) \\
& \mathbf{U}_{N, 1}^{\nu} & \vdots \\
-\int_{\mathbb{R}} p_{0}(\lambda) d \nu(\lambda) & \cdots & -\int_{\mathbb{R}} p_{N-1}(\lambda) d \nu(\lambda) & \int_{\mathbb{R}} p_{N-1}(\lambda) d \nu(\lambda) \\
& & 0
\end{array}\right] .
$$

\section{2 for Real Asymmetric Ensembles}

The corresponding identity for real asymmetric ensembles is given in [17]. This can be written down as follows: Given a measure $\nu_{1}$ on $\mathbb{R}$ and a measure $\nu_{2}$ on $\mathbb{C} \backslash \mathbb{R}$ which is invariant under complex conjugation, define $\nu=\nu_{1}+\nu_{2}$ and set

$$
Z_{N}^{\nu}=\sum_{\substack{(L, M) \\ L+2 M=N}} \frac{1}{2^{M} M ! L !} \int_{\mathbb{R}^{L}} \int_{\mathbb{C}^{M}}|\Delta(\boldsymbol{\alpha} \vee \boldsymbol{\beta} \curlyvee \overline{\boldsymbol{\beta}})| d \nu_{1}^{L}(\boldsymbol{\alpha}) d \nu_{2}^{M}(\boldsymbol{\beta}) .
$$

If $d \nu_{1}=w d \mu_{1}$ and $d \nu_{2}(\beta)=|w|^{2} d \mu_{2}$ for some function $w: \mathbb{C} \rightarrow[0, \infty)$, then $Z_{N}^{\nu}$ is the normalizing constant for the real asymmetric ensemble with weight function $w$. When $N$ is even,

$$
Z_{N}^{\nu}=\operatorname{Pf} \mathbf{U}_{N}^{\nu},
$$

where $\mathbf{U}_{N}^{\nu}$ is the $N \times N$ antisymmetric matrix given by

$$
\mathbf{U}_{N}^{\nu}=\left[\int_{\mathbb{R}^{2}} p_{j}(\alpha) p_{k}(\gamma) \operatorname{sgn}(\gamma-\alpha) d \nu_{1}(\gamma) d \nu_{1}(\alpha)-2 i \int_{\mathbb{C}} p_{j}(\beta) p_{k}(\bar{\beta}) \operatorname{sgn} \operatorname{Im}(\beta) d \nu_{2}(\beta)\right]_{j, k=0}^{N-1}
$$

and when $N$ is odd, $Z_{N}^{\nu}=\operatorname{Pf} \mathbf{W}_{N}^{\nu}$ where

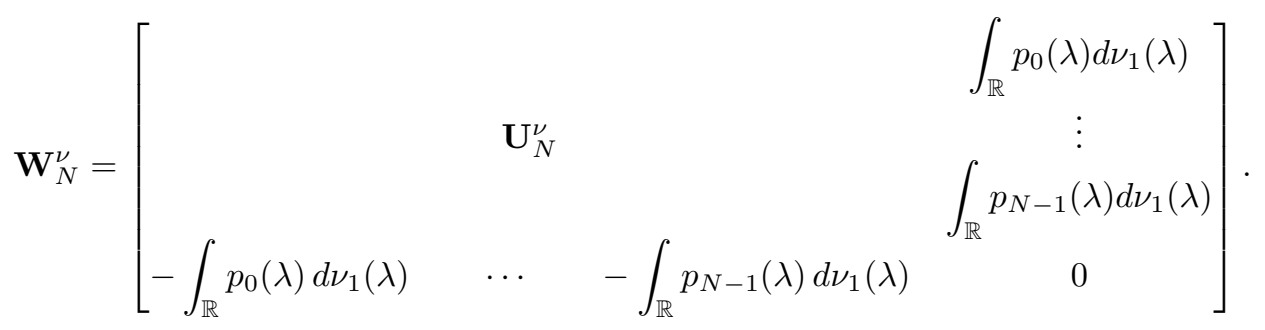

\section{Correlation Functions in terms of Partition Functions}

From here forward we will limit our attention to $\beta=1$ Hermitian ensembles and real asymmetric ensembles. We will drop the subscripts on all relevant quantities for these ensembles so that, for instance, $Z^{\nu}$ represents both $Z_{N, 1}^{\nu}$ and $Z_{N}^{\nu}$; which partition function is being represented will be clear from context. This will allow us to treat these cases simultaneously at the most technical part of the proof.

We will ultimately be interested in the case when $N$ is odd, but the results in this section are equally valid for $N$ even. 


\subsection{For Hermitian Ensembles}

We first consider the case of $w 1 \mathrm{E}$. Let $T>N$ be an even integer, $y_{1}, y_{2}, \ldots, y_{T} \in \mathbb{R}$ and suppose $c_{1}, c_{2}, \ldots, c_{T}$ are indeterminants. We define the measure

$$
d \eta(\lambda)=\sum_{t=1}^{T} c_{t} w\left(y_{t}\right) d \delta\left(\lambda-y_{t}\right)
$$

where $\delta$ is the probability measure with unit mass at $x=0$. We also define the measure $\nu$ by $d \nu=w d \mu . Z^{\eta+\nu} / Z^{\nu}$ is the generating function for the correlation functions of $w 1 \mathrm{E}$; this is the content of the following lemma.

Lemma 3.1. Given an integer $n>0$ we define $\underline{n}=\{1,2, \ldots, n\}$.

$$
\frac{Z^{\eta+\nu}}{Z^{\nu}}=1+\sum_{n=1}^{T} \sum_{\mathfrak{t}: \underline{\underline{n}} \nearrow_{\underline{T}}}\left\{\prod_{j=1}^{n} c_{\mathfrak{t}(j)}\right\} R_{n}\left(y_{\mathfrak{t}(1)}, \ldots, y_{\mathfrak{t}(n)}\right)
$$

The proof of this lemma follows easily from the definitions of $Z^{\eta+\nu}$ and $R_{n}$; details can be found in $[3]$.

\subsection{For Real Asymmetric Ensemble}

For the real asymmetric ensembles with weight function $w$ we suppose $U$ and $V$ are even integers greater than $N$ and set

$$
d \eta(\lambda)=\sum_{u=1}^{U} a_{u} w\left(x_{u}\right) d \delta\left(\zeta-x_{u}\right)+\sum_{v=1}^{V} b_{v}\left(w\left(z_{v}\right) d \delta\left(\zeta-z_{v}\right)+w\left(\bar{z}_{v}\right) \delta\left(\zeta-\bar{z}_{v}\right)\right)
$$

where $x_{1}, \ldots, x_{U} \in \mathbb{R}, z_{1}, \ldots, z_{V} \in \mathbb{C} \backslash \mathbb{R}$ and $a_{1}, \ldots, a_{U}, b_{1}, \ldots, b_{V}$ are indeterminants. If $\nu=\nu_{1}+\nu_{2}$ is the measure with $d \nu_{1}=w d \mu_{1}$ and $d \nu_{2}=|w|^{2} d \mu_{2}$, then $Z^{\eta+\nu} / Z^{\nu}$ generates the correlation functions of the corresponding real asymmetric ensemble.

\section{Lemma 3.2.}

$$
\left.\frac{Z^{\eta+\nu}}{Z^{\nu}}=\sum_{\substack{(\ell, m) \\ \ell+2 m \leq N}} \sum_{\mathfrak{u}: \underline{\ell} \nearrow \underline{\underline{U}} \mathfrak{v}: \underline{m} \nearrow \underline{\underline{V}}} \sum_{j=1}^{\ell} a_{\mathfrak{u}(\ell)} \prod_{k=1}^{m} b_{\mathfrak{v}(k)}\right\} R_{\ell, m}\left(x_{\mathfrak{u}(1)}, \ldots, x_{\mathfrak{u}(\ell)}, z_{\mathfrak{v}(1)}, \ldots, z_{\mathfrak{v}(m)}\right)
$$

where by convention we will take

$$
\sum_{\mathfrak{u}: \underline{\underline{0}} \nearrow \underline{N} \underline{j}=1} \prod_{\mathfrak{u}=1}^{0} a_{\mathfrak{u}(j)}=\sum_{\mathfrak{v}: \underline{\underline{0}} \nearrow_{\underline{N}}} \prod_{k=1}^{0} b_{\mathfrak{v}(j)}=1 .
$$

We remark that Lemma 3.1 follows from Lemma 3.2 by setting $b_{1}=\cdots=b_{V}=0$. The proof of Lemma 3.2 is found in [3] and follows directly from the definitions of $Z^{\eta+\nu}$ and $R_{\ell, m}$.

\section{Using de Bruijn's Identities}

From here forward we will assume that $N$ is odd. 


\subsection{For Hermitian Ensembles}

Given a measure $\kappa$ on $\mathbb{R}$ we define the operator $\epsilon^{\kappa}: L^{2}(\kappa) \rightarrow L^{2}(\kappa)$ by

$$
\epsilon^{\kappa} f(\lambda)=\frac{1}{2} \int_{\mathbb{R}} f(\xi) \operatorname{sgn}(\lambda-\xi) d \kappa(\lambda) .
$$

Using this we define a skew-symmetric bilinear form on $L^{2}(\kappa)$ given by

$$
\langle f \mid g\rangle^{\kappa}=\int_{\mathbb{R}}\left(f \epsilon^{\kappa} g-g \epsilon^{\kappa} f\right) d \kappa .
$$

Notice that $\mathbf{U}^{\nu}$, as given in (2.1), can also be written as

$$
\mathbf{U}^{\nu}=\left[\left\langle p_{j} \mid p_{k}\right\rangle^{\nu}\right]_{j, k=0}^{N-1},
$$

where, as before $p_{0}, p_{1}, \ldots$ is a sequence of monic polynomials with $\operatorname{deg} p_{n}=n$.

Since $Z^{\eta+\nu} / Z^{\nu}$ generates the correlation functions, we turn now to the investigation of the entries of $\mathbf{W}^{\eta+\nu}$. It will be convenient to set $\widetilde{p}_{n}=w p_{n} ; n=1,2, \ldots$, and to write $\langle f \mid g\rangle=\langle f \mid g\rangle^{\mu}$ and $\epsilon=\epsilon^{\mu}$ (recall $\mu$ is Lebesgue measure on $\mathbb{R}$ ).

$$
\begin{array}{r}
\left\langle p_{j} \mid p_{k}\right\rangle_{\eta+\nu}=\int_{\mathbb{R}^{2}} p_{j}(\lambda) p_{k}(\xi) \operatorname{sgn}(\xi-\lambda) d(\nu+\eta)(\lambda) d(\nu+\eta)(\xi) \\
=\left\langle\widetilde{p}_{j} \mid \widetilde{p}_{k}\right\rangle_{\mu}+2 \sum_{t=1}^{T} c_{t}\left(\widetilde{p}_{j}\left(y_{t}\right) \epsilon \widetilde{p}_{k}\left(y_{t}\right)-\widetilde{p}_{k}\left(y_{t}\right) \epsilon \widetilde{p}_{j}\left(y_{t}\right)\right) \\
\quad-\sum_{t=1}^{T} \sum_{m=1}^{T} c_{t} c_{m} \widetilde{p}_{j}\left(y_{t}\right) \widetilde{p}_{k}\left(y_{m}\right) \operatorname{sgn}\left(y_{m}-y_{t}\right) .
\end{array}
$$

The other entries in $\mathbf{W}^{\eta+\nu}$ are of the form

$$
\int_{\mathbb{R}} p_{j}(\lambda) d(\eta+\nu)(\lambda)=\int_{\mathbb{R}} \widetilde{p}_{j}(\lambda) d \mu(\lambda)+\sum_{t=1}^{T} c_{t} \widetilde{p}_{j}\left(y_{t}\right)
$$

\subsection{For Real Asymmetric Ensemble}

First we extend the definition of the sgn function to $\mathbb{C}$ by specifying that $\operatorname{sgn}(z)=0$ if $z \notin \mathbb{R}$. Next, given a measure $\kappa=\kappa_{1}+\kappa_{2}$ on $\mathbb{C}$ we define the operator $\epsilon^{\kappa}: L^{2}(\kappa) \rightarrow L^{2}(\kappa)$ by

$$
\epsilon^{\kappa} f(\lambda)= \begin{cases}\frac{1}{2} \int_{\mathbb{R}} f(\xi) \operatorname{sgn}(\lambda-\xi) d \kappa_{1}(\lambda) & \lambda \in \mathbb{R} ; \\ -i \operatorname{sgn} g(\bar{\lambda}) \operatorname{sgn} \operatorname{Im}(\lambda) & \lambda \in \mathbb{C} \backslash \mathbb{R} .\end{cases}
$$

As in the Hermitian case, we define a skew-symmetric bilinear form on $L^{2}(\kappa)$ by

$$
\langle f \mid g\rangle^{\kappa}=\int_{\mathbb{C}}\left(f \epsilon^{\kappa} g-g \epsilon^{\kappa} f\right) d \kappa .
$$

As before we set $\epsilon$ and $\langle f \mid g\rangle$ for $\epsilon^{\mu}$ and $\langle f \mid g\rangle^{\mu}$, where in the context of real asymmetric ensembles, $\mu=\mu_{1}+\mu_{2}$. 
A superficial change of notation will bring the entries of $\mathbf{W}^{\eta+\nu}$ into a form identical to the Hermitian case. We set $T=U+V$, rename the indeterminants $a_{1}, \ldots, a_{U}, b_{1}, \ldots, b_{V}$ to $c_{1}, c_{2}, \ldots, c_{T}$ and rename $x_{1}, \ldots, x_{U}, z_{1}, \ldots, z_{V}$ to $y_{1}, y_{2}, \ldots, y_{T}$. If we also define

$$
d \widehat{\delta}(\lambda-\gamma)= \begin{cases}d \delta(\lambda-\gamma) & \gamma \in \mathbb{R} ; \\ d \delta(\lambda-\gamma)+d \delta(\lambda-\bar{\gamma}) & \gamma \in \mathbb{C} \backslash \mathbb{R} .\end{cases}
$$

It follows that we may write

$$
d \eta(\lambda)=\sum_{t=1}^{T} c_{t} w\left(x_{t}\right) d \widehat{\delta}\left(\lambda-y_{t}\right)
$$

and, as in the Hermitian ensemble case,

$$
\begin{aligned}
\left\langle p_{j} \mid p_{k}\right\rangle_{\eta+\nu}=\left\langle\widetilde{p}_{j} \mid \widetilde{p}_{k}\right\rangle_{\mu}+2 \sum_{t=1}^{T} c_{t}\left(\widetilde{p}_{j}\left(y_{t}\right) \epsilon \widetilde{p}_{k}\left(y_{t}\right)-\widetilde{p}_{k}\left(y_{t}\right) \epsilon \widetilde{p}_{j}\left(y_{t}\right)\right) & \\
& -\sum_{t=1}^{T} \sum_{m=1}^{T} c_{t} c_{m} \widetilde{p}_{j}\left(y_{t}\right) \widetilde{p}_{k}\left(y_{m}\right) \operatorname{sgn}\left(y_{m}-y_{t}\right),
\end{aligned}
$$

and

$$
\int_{\mathbb{R}} p_{j}(\lambda) d(\eta+\nu)(\lambda)=\int_{\mathbb{R}} \widetilde{p}_{j}(\lambda) d \mu(\lambda)+\sum_{t=1}^{T} c_{t} \widetilde{p}_{j}\left(y_{t}\right) .
$$

\section{The Method of Tracy and Widom}

Since we have written $Z^{\eta+\nu} / Z^{\nu}$ in a uniform manner for both Hermitian ensembles and real asymmetric ensembles we may, at least for the moment, analyze both cases simultaneously.

We define $\mathbf{A}$ to be the $N+1 \times 2 T$ matrix given by

$$
\mathbf{A}=\left[\begin{array}{ccccc}
\sqrt{2 c_{1}} \widetilde{p}_{0}\left(y_{1}\right) & \sqrt{2 c_{1}} \epsilon \widetilde{p}_{0}\left(y_{1}\right) & & \sqrt{2 c_{T}} \widetilde{p}_{0}\left(y_{T}\right) & \sqrt{2 c_{T}} \epsilon \widetilde{p}_{0}\left(y_{T}\right) \\
\sqrt{2 c_{1}} \widetilde{p}_{1}\left(y_{1}\right) & \sqrt{2 c_{1}} \epsilon \widetilde{p}_{1}\left(y_{1}\right) & \cdots & \sqrt{2 c_{T}} \widetilde{p}_{1}\left(y_{T}\right) & \sqrt{2 c_{T}} \epsilon \widetilde{p}_{1}\left(y_{T}\right) \\
& \vdots & \ddots & \vdots & \\
\sqrt{2 c_{1} \widetilde{p}_{N-1}\left(y_{1}\right)} & \sqrt{2 c_{1}} \epsilon \widetilde{p}_{N-1}\left(y_{1}\right) & \cdots & \sqrt{2 c_{T}} \widetilde{p}_{N-1}\left(y_{T}\right) & \sqrt{2 c_{T}} \epsilon \widetilde{p}_{N-1}\left(y_{T}\right) \\
0 & \chi_{\mathbb{R}}\left(y_{1}\right) \sqrt{c_{1} / 2} & & 0 & \chi_{\mathbb{R}}\left(y_{T}\right) \sqrt{c_{T} / 2}
\end{array}\right],
$$

where $\chi_{\mathbb{R}}$ is the characteristic function of $\mathbb{R}$. That is,

$$
\begin{array}{rlr}
\mathbf{A}_{n, 2 t-1}=\sqrt{2 c_{t}} \widetilde{p}_{n}\left(y_{t}\right), & n=0,1, \ldots, N-1 & t=1,2, \ldots, T ; \\
\mathbf{A}_{n, 2 t}=\sqrt{2 c_{t}} \epsilon \widetilde{p}_{n}\left(y_{t}\right), & n=0,1, \ldots, N-1 & t=1,2, \ldots, T,
\end{array}
$$

and

$$
\begin{array}{ll}
\mathbf{A}_{N, 2 t-1}=0, & t=1,2, \ldots, T \\
\mathbf{A}_{N, 2 t}=\chi_{\mathbb{R}}\left(y_{t}\right) \sqrt{c_{t} / 2}, & t=1,2, \ldots, T .
\end{array}
$$


and set $\mathbf{J}$ to be the $2 T \times 2 T$ matrix,

$$
\mathbf{J}=\left[\begin{array}{ccccc}
0 & 1 & & & \\
-1 & 0 & & & \\
& & \ddots & & \\
& & & 0 & 1 \\
& & & -1 & 0
\end{array}\right]
$$

It follows that,

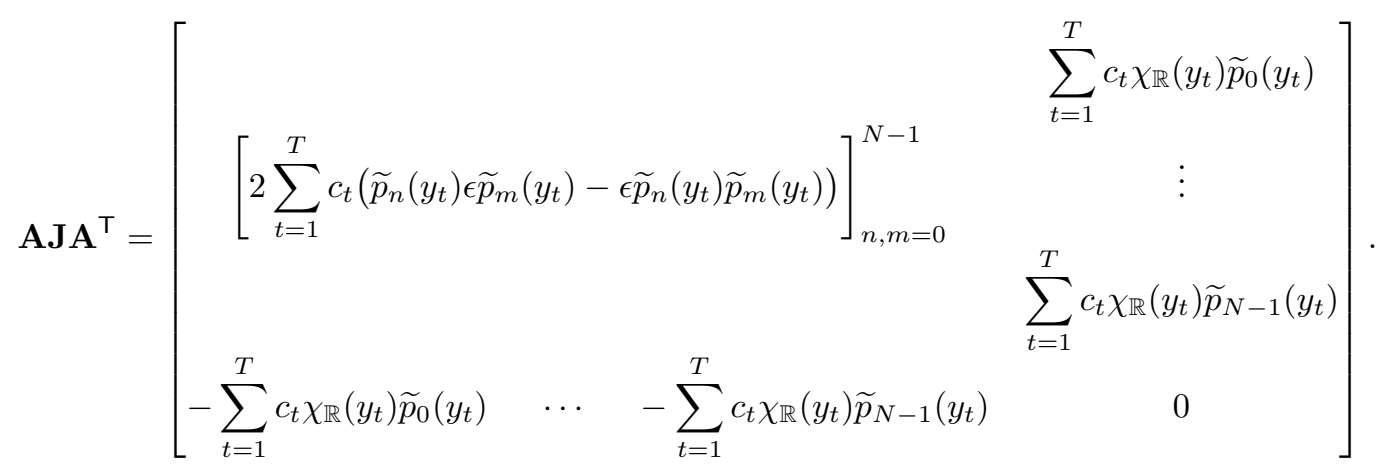

Next, we define the $2 T \times 2 T$ matrix

$$
\mathbf{E}=\left[\begin{array}{cc}
\frac{\sqrt{c_{t} c_{u}}}{2} \operatorname{sgn}\left(y_{u}-y_{t}\right) & 0 \\
0 & 0
\end{array}\right]_{t, u=1}^{T}
$$

so that,

$$
\mathbf{E}_{2 t-1,2 u-1}=\frac{\sqrt{c_{t} c_{u}}}{2} \operatorname{sgn}\left(y_{u}-y_{t}\right) ; \quad t, u=1,2, \ldots, T,
$$

and all other entries of $\mathbf{E}$ are equal to 0 . An easy computation reveals

$$
\left(\mathbf{A E A} \mathbf{A}^{\top}\right)_{n, m}=\sum_{u=1}^{T} \sum_{t=1}^{T} c_{u} c_{t} \widetilde{p}_{m}\left(y_{u}\right) \widetilde{p}_{n}\left(y_{t}\right) \operatorname{sgn}\left(y_{u}-y_{t}\right) ; \quad n, m=0,1, \ldots, N-1,
$$

and all entries in the last row and column of $\left(\mathbf{A E A} \mathbf{A}^{\top}\right)$ are 0 . That is,

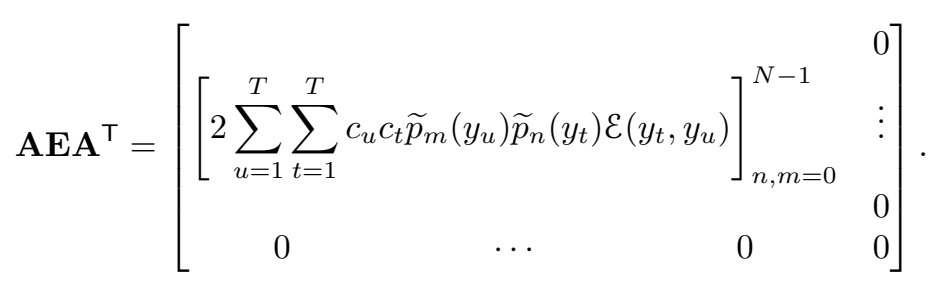

It follows that

$$
\mathbf{W}^{\eta+\nu}=\mathbf{W}^{\nu}+\mathbf{A} \mathbf{J} \mathbf{A}^{\top}-\mathbf{A} \mathbf{E} \mathbf{A}^{\top} .
$$

And, if we define the $N+1 \times N+1$ matrices $\mathbf{C}^{-\boldsymbol{T}}=\mathbf{W}^{\nu}$ and $\mathbf{B}=-\mathbf{J}+\mathbf{E}$, then

$$
\frac{Z^{\nu+\eta}}{Z^{\nu}}=\frac{\operatorname{Pf} \mathbf{W}^{\nu+\eta}}{\operatorname{Pf} \mathbf{W}^{\nu}}=\frac{\operatorname{Pf}\left(\mathbf{C}^{-\boldsymbol{\top}}-\mathbf{A B} \mathbf{A}^{\top}\right)}{\operatorname{Pf} \mathbf{C}^{-\top}} .
$$


We are now in position to use the identity

$$
\frac{\operatorname{Pf}\left(\mathbf{C}^{-\boldsymbol{T}}-\mathbf{A B} \mathbf{A}^{\top}\right)}{\operatorname{Pf} \mathbf{C}^{-\top}}=\frac{\operatorname{Pf}\left(\mathbf{B}^{-\top}-\mathbf{A}^{\top} \mathbf{C A}\right)}{\operatorname{Pf} \mathbf{B}^{-\top}},
$$

and an easy calculation shows that

$$
\mathbf{B}^{-\mathbf{T}}=-\mathbf{J}-\mathbf{E}^{\prime}
$$

where $\mathbf{E}^{\prime}$ is the $2 T \times 2 T$ matrix,

$$
\mathbf{E}^{\prime}=\left[\begin{array}{lc}
0 & 0 \\
0 & \frac{\sqrt{c_{t} c_{u}}}{2} \\
\operatorname{sgn}\left(y_{u}-y_{t}\right)
\end{array}\right]_{t, u=1}^{T} .
$$

It is also easily verified that $\operatorname{Pf} \mathbf{B}^{-\top}=(-1)^{T}$. Thus,

$$
\frac{Z^{\nu+\eta}}{Z^{\nu}}=(-1)^{T} \operatorname{Pf}\left(-\mathbf{J}-\mathbf{E}^{\prime}-\mathbf{A}^{\top} \mathbf{C A}\right)=\operatorname{Pf}\left(\mathbf{J}+\mathbf{E}^{\prime}+\mathbf{A}^{\top} \mathbf{C A}\right) .
$$

We now compute the entries of $\mathbf{A}^{\top} \mathbf{C A}$ :

$$
\begin{gathered}
\left(\mathbf{A}^{\top} \mathbf{C A}\right)_{2 u-1,2 t-1}=2 \sqrt{c_{u} c_{t}} \sum_{n=0}^{N-1} \sum_{m=0}^{N-1} \widetilde{p}_{n}\left(y_{u}\right) \mathbf{C}_{n, m} \widetilde{p}_{m}\left(y_{t}\right) ; \quad u, t=1,2, \ldots, T \\
\left(\mathbf{A}^{\top} \mathbf{C A}\right)_{2 u-1,2 t}=2 \sqrt{c_{u} c_{t}} \sum_{n=0}^{N-1} \sum_{m=0}^{N-1} \widetilde{p}_{n}\left(y_{u}\right) \mathbf{C}_{n, m} \epsilon \widetilde{p}_{m}\left(y_{t}\right)+\sqrt{c_{u} c_{t}} \chi_{\mathbb{R}}\left(y_{t}\right) \sum_{n=0}^{N-1} \widetilde{p}_{n}\left(y_{u}\right) \mathbf{C}_{n, N} \\
u, t=1,2, \ldots, T ; \\
\left(\mathbf{A}^{\top} \mathbf{C A}\right)_{2 u, 2 t-1}=2 \sqrt{c_{u} c_{t}} \sum_{n=0}^{N-1} \sum_{m=0}^{N-1} \epsilon \widetilde{p}_{n}\left(y_{u}\right) \mathbf{C}_{n, m} \widetilde{p}_{m}\left(y_{t}\right)+\sqrt{c_{u} c_{t}} \chi_{\mathbb{R}}\left(y_{u}\right) \sum_{m=0}^{N-1} \mathbf{C}_{N, m} \widetilde{p}_{m}\left(y_{t}\right) ; \\
\left(\mathbf{A}^{\top} \mathbf{C A}\right)_{2 u, 2 t}=\sqrt{c_{u} c_{t}} \chi_{\mathbb{R}}\left(y_{t}\right) \sum_{n=0}^{N-1} \mathbf{C}_{n, N} \epsilon \widetilde{p}_{n}\left(y_{u}\right)+\sqrt{c_{u} c_{t}} \chi_{\mathbb{R}}\left(y_{u}\right) \sum_{m=0}^{N-1} \mathbf{C}_{N, m} \epsilon \widetilde{p}_{m}\left(y_{t}\right) ; \\
+2 \sqrt{c_{u} c_{t}} \sum_{n=0}^{N-1} \sum_{m=0}^{N-1} \epsilon \widetilde{p}_{n}\left(y_{u}\right) \mathbf{C}_{n, m} \epsilon \widetilde{p}_{m}\left(y_{t}\right) ; \quad u, t=1,2, \ldots, T
\end{gathered}
$$

If we define

$$
\begin{aligned}
D S_{N}\left(y, y^{\prime}\right) & =2 \sum_{n=0}^{N-1} \sum_{m=0}^{N-1} \widetilde{p}_{n}(y) \mathbf{C}_{n, m} \widetilde{p}_{m}\left(y^{\prime}\right) \\
S_{N}\left(y, y^{\prime}\right) & =2 \sum_{n=0}^{N-1} \sum_{m=0}^{N-1} \widetilde{p}_{n}(y) \mathbf{C}_{n, m} \epsilon \widetilde{p}_{m}\left(y^{\prime}\right)+\chi_{\mathbb{R}}\left(y^{\prime}\right) \sum_{n=0}^{N-1} \widetilde{p}_{n}(y) \mathbf{C}_{n, N} ; \\
S_{N} I\left(y, y^{\prime}\right) & =2 \sum_{n=0}^{N-1} \sum_{m=0}^{N-1} \epsilon \widetilde{p}_{n}(y) \mathbf{C}_{n, m} \epsilon \widetilde{p}_{m}\left(y^{\prime}\right) \\
& +\sum_{n=0}^{N-1} \mathbf{C}_{n, N}\left(\chi_{\mathbb{R}}\left(y^{\prime}\right) \epsilon \widetilde{p}_{n}(y)-\chi_{\mathbb{R}}(y) \epsilon \widetilde{p}_{n}\left(y^{\prime}\right)\right),
\end{aligned}
$$


then

$$
\mathbf{A}^{\top} \mathbf{C A}=\left[\sqrt{c_{u} c_{t}}\left[\begin{array}{ll}
D S_{N}\left(y_{u}, y_{t}\right) & S_{N}\left(y_{u}, y_{t}\right) \\
-S_{N}\left(y_{t}, y_{u}\right) & S_{N} I\left(y_{u}, y_{t}\right)
\end{array}\right]\right]_{u, t=1}^{T},
$$

and, if we define the $2 \times 2$ matrix kernel $K_{N}: \mathbb{C} \times \mathbb{C} \rightarrow \mathbb{C}^{2 \times 2}$ by

$$
K_{N}\left(y, y^{\prime}\right)=\left[\begin{array}{cc}
D S_{N}\left(y, y^{\prime}\right) & S_{N}\left(y, y^{\prime}\right) \\
-S_{N}\left(y, y^{\prime}\right) & S_{N} I\left(y, y^{\prime}\right)+\frac{1}{2} \operatorname{sgn}\left(y^{\prime}-y\right)
\end{array}\right],
$$

and the $2 T \times 2 T$ matrix $\mathbf{K}$ by

$$
\mathbf{K}=\left[\sqrt{c_{u} c_{t}} K_{N}\left(y_{u}, y_{t}\right)\right]_{u, t=1}^{T}
$$

then from (5.1), (5.2), (5.3) we see that

$$
\frac{Z^{\nu+\eta}}{Z^{\nu}}=\operatorname{Pf}(\mathbf{J}+\mathbf{K})
$$

\section{Recovering the Correlation Functions}

The final step in the derivation of the correlation functions is to expand $\operatorname{Pf}(\mathbf{J}+\mathbf{K})$ using the identity

$$
\operatorname{Pf}(\mathbf{J}+\mathbf{K})=1+\sum_{n=1}^{T} \sum_{\mathfrak{t}: \underline{\underline{ }} \nearrow_{\underline{T}}} \operatorname{Pf} \mathbf{K}_{\mathfrak{t}},
$$

where $\mathbf{K}_{\mathbf{t}}$ is the $2 n \times 2 n$ minor of $\mathbf{K}$ given by

$$
\mathbf{K}_{\mathfrak{t}}=\left[\sqrt{c_{\mathfrak{t}(j)} C_{\mathfrak{t}(k)}} K_{N}\left(y_{\mathfrak{t}(j)}, y_{\mathfrak{t}(k)}\right)\right]_{j, k=1}^{n} .
$$

A Proof of this identity can be found in [21] or [18].

\subsection{For Hermitian Ensembles}

Applying (6.1) to (5.4) and using the fact that

$$
\operatorname{Pf} \mathbf{K}_{\mathfrak{t}, \mathfrak{t}}=\left\{\prod_{m=1}^{n} c_{\mathfrak{t}(m)}\right\} \operatorname{Pf}\left[K_{N}\left(y_{\mathfrak{t}(j)}, y_{\mathfrak{t}(k)}\right)\right]_{j, k=1}^{n},
$$

we find that

$$
\frac{Z^{\nu+\eta}}{Z^{\nu}}=1+\sum_{n=1}^{T} \sum_{\mathfrak{t}: \underline{n} \nearrow \underline{T}}\left\{\prod_{m=1}^{n} c_{\mathfrak{t}(m)}\right\} \operatorname{Pf}\left[K_{N}\left(y_{\mathfrak{t}(j)}, y_{\mathfrak{t}(k)}\right)\right]_{j, k=1}^{n} .
$$

This together with Lemma 3.1 implies that

$$
R_{n}(\mathbf{y})=\operatorname{Pf}\left[K_{N}\left(y_{j}, y_{k}\right)\right]_{j, k=1}^{n} .
$$




\subsection{For Real Asymmetric Ensembles}

Given $\mathfrak{u}: \underline{\ell} \nearrow \underline{U}$ and $\mathfrak{v}: \underline{m} \nearrow \underline{V}$, we define $\mathfrak{u} \vee \mathfrak{v}: \underline{\ell+m} \nearrow \underline{U+V}$ by

$$
(\mathfrak{u} \vee \mathfrak{v})(j)= \begin{cases}\mathfrak{u}(j) & j \leq \ell \\ \ell+\mathfrak{v}(j) & j>\ell\end{cases}
$$

If $\ell+m=n$, then each $\mathfrak{t}: \underline{n} \nearrow \underline{T}$ can be written as $\mathfrak{u} \vee \mathfrak{v}$ for some $\mathfrak{u}: \underline{\ell} \nearrow \underline{U}$ and $\mathfrak{v}: \underline{m} \nearrow \underline{V}$. (This does not preclude the possibility that $\mathfrak{t}=\mathfrak{u}$ or $\mathfrak{t}=\mathfrak{v}$ ). Using (6.1) we find

$$
\operatorname{Pf}(\mathbf{J}+\mathbf{K})=\sum_{\substack{\ell, m) \\ \ell \leq U, m \leq V}} \sum_{\mathfrak{u}: \underline{\ell} \nearrow \underline{\underline{U}} \mathfrak{v}: \underline{m} \nearrow \underline{\underline{V}}} \sum \operatorname{Pf} \mathbf{K}_{\mathfrak{u} \vee \mathfrak{v}}
$$

where we take the Pfaffian of an empty matrix (i.e. when $\ell$ and $m$ equal 0 ) to be 1 . It is straightforward, if technical, to show that

$$
\operatorname{Pf} \mathbf{K}_{\mathfrak{u} \vee \mathfrak{v}}=\left\{\prod_{j=1}^{\ell} a_{\mathfrak{u}(j)} \prod_{k=1}^{m} b_{\mathfrak{v}(k)}\right\} \operatorname{Pf}\left[\begin{array}{ll}
K_{N}\left(x_{\mathfrak{u}(j)}, x_{\mathfrak{u}\left(j^{\prime}\right)}\right) & K_{N}\left(x_{\mathfrak{u}(j)}, z_{\mathfrak{v}\left(k^{\prime}\right)}\right) \\
K_{N}\left(z_{\mathfrak{v}(k)}, x_{\mathfrak{u}\left(k^{\prime}\right)}\right) & K_{N}\left(z_{\mathfrak{v}(k)}, z_{\mathfrak{v}\left(k^{\prime}\right)}\right)
\end{array}\right]_{\substack{j, j^{\prime}=1, \ldots, \ell \\
k, k^{\prime}=1, \ldots, m}} .
$$

Thus, from (5.4),

$$
\frac{Z^{\nu+\eta}}{Z^{\nu}}=\sum_{\substack{(\ell, m) \\
0 \leq \ell+m \leq T}} \sum_{\mathfrak{u}: \underline{\ell} \nearrow \underline{\underline{U}} \mathfrak{v}: \underline{\underline{m}} \nearrow \underline{\underline{V}}}\left\{\prod_{j=1}^{\ell} a_{\mathfrak{u}(j)} \prod_{k=1}^{m} b_{\mathfrak{v}(k)}\right\} \operatorname{Pf}\left[\begin{array}{ll}
K_{N}\left(x_{\mathfrak{u}(j)}, x_{\mathfrak{u}\left(j^{\prime}\right)}\right) & K_{N}\left(x_{\mathfrak{u}(j)}, z_{\mathfrak{v}\left(k^{\prime}\right)}\right) \\
K_{N}\left(z_{\mathfrak{v}(k)}, x_{\mathfrak{u}\left(k^{\prime}\right)}\right) & K_{N}\left(z_{\mathfrak{v}(k)}, z_{\mathfrak{v}\left(k^{\prime}\right)}\right)
\end{array}\right]
$$

This result and Lemma 3.2 imply that

$$
R_{\ell, m}(\mathbf{x}, \mathbf{z})=\operatorname{Pf}\left[\begin{array}{ll}
K_{N}\left(x_{j}, x_{j^{\prime}}\right) & K_{N}\left(x_{j}, z_{k^{\prime}}\right) \\
K_{N}\left(z_{k}, x_{j^{\prime}}\right) & K_{N}\left(z_{k}, z_{k^{\prime}}\right)
\end{array}\right]_{\substack{j, j^{\prime}=1, \ldots, \ell \\
k, k^{\prime}=1, \ldots, m}} .
$$

\section{Simplifying the Matrix Kernel}

On first inspection, the kernel $K_{N}$ is dependent on $\left(p_{0}, p_{2}, \ldots, p_{N-1}\right)$. In fact, $K_{N}$ can be shown to be independent of this family of moni polynomials - though a wise choice may simplify the representation of $K_{N}$. In this section we suppose the existence of a complete monic family of polynomials $\mathbf{q}=\left(q_{0}, q_{1}, \ldots, q_{N-1}\right)$ such that

$$
\left\langle\widetilde{q}_{2 n} \mid \widetilde{q}_{2 m-1}\right\rangle_{\nu}=-\left\langle\widetilde{q}_{2 m-1} \mid \widetilde{q}_{2 n}\right\rangle_{\nu}=\delta_{n, m} r_{n} \quad n=0,1, \ldots, J,
$$

where $J=(N-1) / 2-1$. Here $\mathbf{r}=\left(r_{1}, r_{2}, \ldots, r_{J}\right)$ are referred to the normalizations of $\mathbf{q}$. We also define

$$
s_{n}=\int_{\mathbb{R}} \widetilde{q}_{n}(\alpha) d \nu_{\mathbb{R}}(\alpha), \quad n=0,1, \ldots, N-1 .
$$

Clearly $\mathbf{q}, \mathbf{r}$ and $\mathbf{s}$ are dependent on $w$. 
Using these definitions, $\mathbf{W}^{\nu}$ becomes

$$
\mathbf{W}^{\nu}=\left[\begin{array}{ccccccccc}
0 & r_{0} & & & & & & 0 & s_{0} \\
-r_{0} & 0 & & & & & & 0 & s_{1} \\
& & 0 & r_{1} & & & & 0 & s_{2} \\
& & -r_{1} & 0 & & & & 0 & s_{3} \\
& & & & \ddots & & & \vdots & \vdots \\
& & & & & 0 & r_{J} & 0 & s_{N-3} \\
& & & & & -r_{J} & 0 & 0 & s_{N-2} \\
0 & 0 & 0 & 0 & \cdots & 0 & 0 & 0 & s_{N-1} \\
-s_{0} & -s_{1} & -s_{2} & -s_{3} & \cdots & -s_{N-3} & -s_{N-2} & -s_{N-1} & 0
\end{array}\right] .
$$

An easy computation reveals,

$$
Z^{\nu}=\operatorname{Pf} \mathbf{W}^{\nu}=s_{N-1} \prod_{j=1}^{J} r_{j} .
$$

In order to present the entries of $K_{N}$ in their most simplified form, we need to invert $\mathbf{W}^{\nu}$. Recall that $\mathbf{C}=\left(\mathbf{W}^{\nu}\right)^{-\top}$. Explicitly, $\mathbf{C}$ is given by

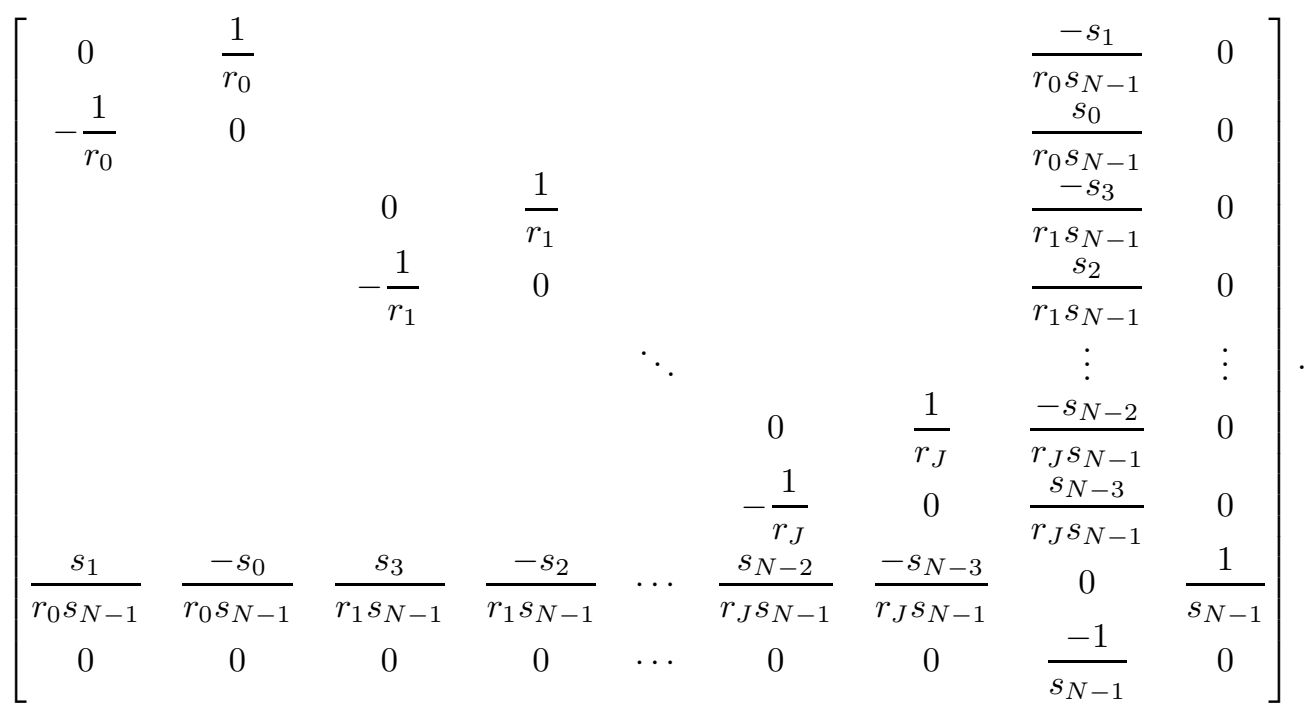

At the entry level,

$$
\begin{aligned}
& \mathbf{C}_{2 j, 2 m+1}=-\mathbf{C}_{2 j+1,2 m}=\frac{\delta_{j, m}}{r_{j}}, \quad \mathbf{C}_{2 j, 2 m}=\mathbf{C}_{2 j+1,2 m+1}=0, \quad j, m=0,1, \ldots, J ; \\
& \mathbf{C}_{2 j, N-1}=-\mathbf{C}_{N-1,2 j}=-\frac{s_{2 j+1}}{r_{j} s_{N-1}}, \quad \mathbf{C}_{2 j+1, N-1}=-\mathbf{C}_{N-1,2 j+1}=\frac{s_{2 j}}{r_{j} s_{N-1}}, \quad j=0,1, \ldots, J ;
\end{aligned}
$$

and

$$
\mathbf{C}_{N, m}=-\mathbf{C}_{m, N}=-\frac{\delta_{m+1, N}}{s_{N-1}}, \quad m=0,1 \ldots, N
$$


It follows that

$$
\begin{gathered}
D S_{N}\left(y, y^{\prime}\right)=2 \sum_{j=0}^{J} \frac{\widetilde{q}_{2 j}(y) \widetilde{q}_{2 j+1}\left(y^{\prime}\right)-\widetilde{q}_{2 j+1}(y) \widetilde{q}_{2 j}\left(y^{\prime}\right)}{r_{j}} \\
+\frac{2 \widetilde{q}_{N-1}(y)}{s_{N-1}} \sum_{j=0}^{J} \frac{s_{2 j+1} \widetilde{q}_{2 j}\left(y^{\prime}\right)-s_{2 j} \widetilde{q}_{2 j+1}\left(y^{\prime}\right)}{r_{j}}+\frac{2 \widetilde{q}_{N-1}\left(y^{\prime}\right)}{s_{N-1}} \sum_{j=0}^{J} \frac{s_{2 j} \widetilde{q}_{2 j+1}(y)-s_{2 j+1} \widetilde{q}_{2 j}(y)}{r_{j}}, \\
S_{N}\left(y, y^{\prime}\right)=2 \sum_{j=0}^{J} \frac{\widetilde{q}_{2 j}(y) \widetilde{\epsilon} q_{2 j+1}\left(y^{\prime}\right)-\widetilde{q}_{2 j+1}(y) \epsilon \widetilde{q}_{2 j}\left(y^{\prime}\right)}{r_{j}} \\
+\frac{2 \widetilde{q}_{N-1}(y)}{s_{N-1}} \sum_{j=0}^{J} \frac{s_{2 j+1} \epsilon \widetilde{q}_{2 j}\left(y^{\prime}\right)-s_{2 j} \tilde{\epsilon} q_{2 j+1}\left(y^{\prime}\right)}{r_{j}} \\
+\frac{2 \epsilon \widetilde{q}_{N-1}\left(y^{\prime}\right)}{s_{N-1}} \sum_{j=0}^{J} \frac{s_{2 j} \widetilde{q}_{2 j+1}(y)-s_{2 j+1} \widetilde{q}_{2 j}(y)}{r_{j}}+\frac{\widetilde{q}_{N-1}(y) \chi_{\mathbb{R}}\left(y^{\prime}\right)}{s_{N-1}},
\end{gathered}
$$

and

$$
\begin{gathered}
S_{N} I\left(y, y^{\prime}\right)=2 \sum_{j=0}^{J} \frac{\epsilon \widetilde{q}_{2 j}(y) \epsilon \widetilde{q}_{2 j+1}\left(y^{\prime}\right)-\epsilon \widetilde{q}_{2 j+1}(y) \epsilon \widetilde{q}_{2 j}\left(y^{\prime}\right)}{r_{j}} \\
+\frac{2 \epsilon \widetilde{q}_{N-1}(y)}{s_{N-1}} \sum_{j=0}^{J} \frac{s_{2 j+1} \epsilon \widetilde{q}_{2 j}\left(y^{\prime}\right)-s_{2 j} \epsilon \widetilde{q}_{2 j+1}\left(y^{\prime}\right)}{r_{j}} \\
+\frac{2 \epsilon \widetilde{q}_{N-1}\left(y^{\prime}\right)}{s_{N-1}} \sum_{j=0}^{J} \frac{s_{2 j} \epsilon \widetilde{q}_{2 j+1}(y)-s_{2 j+1} \epsilon \widetilde{q}_{2 j}(y)}{r_{j}}+\frac{\epsilon \widetilde{q}_{N-1}(y) \chi_{\mathbb{R}}\left(y^{\prime}\right)-\epsilon \widetilde{q}_{N-1}\left(y^{\prime}\right) \chi_{\mathbb{R}}(y)}{s_{N-1}},
\end{gathered}
$$

\section{References}

[1] M. Adler, P. J. Forrester, T. Nagao, and P. van Moerbeke. Classical skew orthogonal polynomials and random matrices. J. Statist. Phys., 99(1-2):141-170, 2000.

[2] C Andréief. Note sur une relation pour les intégrales défines des produits des fonctions. Mém. Soc. Sci. Bordeaux, 2:1-14, 1883.

[3] Alexei Borodin and Christopher D. Sinclair. Correlation functions of ensembles of asymmetric real matrices, 2007.

[4] Alexei Borodin and Christopher D. Sinclair. The Ginibre ensemble of real random matrices and its scaling limits, 2008.

[5] N. G. de Bruijn. On some multiple integrals involving determinants. J. Indian Math. Soc. (N.S.), 19:133-151 (1956), 1955.

[6] Freeman J. Dyson. Correlations between eigenvalues of a random matrix. Comm. Math. Phys., 19:235-250, 1970.

[7] Alan Edelman. The probability that a random real Gaussian matrix has $k$ real eigenvalues, related distributions, and the circular law. J. Multivariate Anal., 60(2):203-232, 1997. 
[8] Peter Forrester. Log-gases and random matrices. Book in progress.

[9] Peter Forrester and Anthony Mays. A method to calculate correlation functions for $\beta=1$ random matrices of odd size, 2008 .

[10] Peter J. Forrester and Taro Nagao. Eigenvalue statistics of the real Ginibre ensemble. Phys. Rev. Lett., 99, 2007.

[11] Jean Ginibre. Statistical ensembles of complex, quaternion, and real matrices. $J$. Mathematical Phys., 6:440-449, 1965.

[12] N. Lehmann and H.-J. Sommers. Eigenvalue statistics of random real matrices. Physical Review Letters, 67:941-944, August 1991.

[13] G. Mahoux and M. L. Mehta. A method of integration over matrix variables. IV. J. Physique I, 1(8):1093-1108, 1991.

[14] M. L. Mehta. A note on correlations between eigenvalues of a random matrix. Comm. Math. Phys., 20:245-250, 1971.

[15] Madan Lal Mehta. Random matrices, volume 142 of Pure and Applied Mathematics (Amsterdam). Elsevier/Academic Press, Amsterdam, third edition, 2004.

[16] Eric M. Rains. Correlation functions for symmetrized increasing subsequences, 2000.

[17] Christopher D Sinclair. Averages over Ginibre's ensemble of random real matrices. Int. Math. Res. Not., 2007:1-15, 2007.

[18] Christopher D. Sinclair. The range of multiplicative functions on $\mathbb{C}[x], \mathbb{R}[x]$ and $\mathbb{Z}[x]$. Proc. London Math. Soc., 96(3):697-737, 2008.

[19] Hans-Jurgen Sommers. Symplectic structure of the real Ginibre ensemble, 2007.

[20] Hans-Jürgen Sommers and Waldemar Wieczorek. General eigenvalue correlations for the real Ginibre ensemble. J. Phys. A, 41(40), 2008.

[21] John R. Stembridge. Nonintersecting paths, Pfaffians, and plane partitions. Adv. Math., 83(1):96-131, 1990.

Christopher D. Sinclair

Department of Mathematics, University of Colorado, Boulder CO 80309

email: chris.sinclair@colorado.edu 